\title{
Polymer-Coated Composite Anodes for Efficient and Stable
}

\section{Capacitive Deionization}

X. Gao ${ }^{\mathrm{a}}$, A. Omosebi ${ }^{\mathrm{a}}$, N. Holubowitch ${ }^{\mathrm{a}}$, A. Liu ${ }^{\mathrm{a}, \mathrm{b}}, \mathrm{K} . \mathrm{Ruh}^{\mathrm{a}, \mathrm{c}}$, J. Landon ${ }^{\mathrm{a}, *}$, and K. Liu ${ }^{\mathrm{a}, \mathrm{d},{ }^{*}}$

${ }^{a}$ Center for Applied Energy Research, University of Kentucky, Lexington, Kentucky 40511, USA

${ }^{\text {b }}$ Paul Laurence Dunbar High School, Lexington, Kentucky 40513, USA

c Department of Chemical and Materials Engineering, University of Kentucky, Lexington, Kentucky 40506, USA

${ }^{d}$ Department of Mechanical Engineering, University of Kentucky, Lexington, Kentucky 40506, USA

*James.Landon@uky.edu / Kunlei.Liu@uky.edu

Key words - capacitive deionization, performance degradation, carbon oxidation, effluent $\mathrm{pH}$, polymer-coated carbon 


\begin{abstract}
In the contemporary literature, diminished salt removal in a CDI device is primarily due to carbon oxidation at the anode in aqueous solutions. Therefore, an anion exchange polymer is used to prepare a composite carbon as a CDI anode. Results from repetitive CDI testing shows that more efficient and consistent long-term salt removal is achieved when a flow-through CDI stack is configured with composite anodes compared to polymer-free anodes. Analysis of the effluent $\mathrm{pH}$ and steady-state current indicates that this performance improvement may be due to the minimization of parasitic reactions by shielding of the carbon electrodes with the selective polymer layer coated at the anode.
\end{abstract}

\title{
1 Introduction
}

In the past few decades, various desalination technologies have been developed, among which distillation, reverse osmosis, and electrodialysis are the most commonly known and commercially widespread.[1] Capacitive deionization (CDI) is an alternative and energy-efficient technology for water desalination that utilizes an electrochemical flow cell configured with polarizable porous carbon electrodes to remove ionized salts in a stream with lower molar concentration.[2]

In contemporary CDI literature, diminished salt removal in a CDI device during long-term or repetitive operation is one of the remaining issues needing to be addressed. Performance degradation is primarily accounted for by negative surface charges, e.g., $\mathrm{COO}^{-}$, that form via carbon oxidation in aqueous solutions, leading to the positive shifting of the potential of zero charge $\left(E_{\mathrm{PZC}}\right)$ of the anode.[3, 4] Regardless of the carbon 
electrodes used in these studies, the emergence of inverted behavior is typically observed in the form of salt desorption peaks at the beginning of the charging steps (See Fig. 2(a)), which is detrimental to the equilibrium salt removal capacity for a CDI device.[5, 6] Recently, the formation of these inversion peaks was theoretically described by the modified Donnan model by introducing a term for chemical surface charge.[7] It is becoming increasingly clear that fixed surface chemical charges (or charge barriers) play a critical role in CDI, which is supported in ref. [8-15].

Previous efforts to extend the longevity of a CDI device include the reversal of polarization, the reduction of applied voltages, and the use of selective exchange membranes.[3, 16] In this work, we show that a protective layer prepared from anion exchange polymer powder imbues CDI anodes with oxidation resistance. Results from repetitive charging and discharging of flow-through CDI stacks with and without composite anodes demonstrate the efficacy of the protective polymer layer. Additionally, $\mathrm{pH}$ behavior and the $E_{\mathrm{PZC}}$ are invoked to explain the performance enhancements.

\section{Experimental}

\subsection{Electrode Preparation and Characterizations}

The polymer casting solution was prepared using anion exchange powders, n-methyl-2pyrrolidone (NMP) (Sigma-Aldrich), and polyvinylidene fluoride (PVDF) (SigmaAldrich) with a mixture ratio of 12:20:1 by weight. The anion exchange powder was prepared from a type 1 strong base anion resin with trimethylamine functional groups (chloride form, SBG1-F, ResinTech, Inc.) in a planetary mill (Fritsch Pulverisette). The 
resulting solution was brush-painted onto both sides of pristine Spectracarb 2225 carbon cloth (Pr-SC) (Engineered Fibers Technology, LLC), followed by heat treatment at $150^{\circ} \mathrm{C}$ for 30 min under vacuum to remove the solvent and set the polymer. The resulting anionic polymer modified carbon was termed A-SC.

Surface morphology was characterized using a scanning electron microscope (SEM) (S-4800, Hitachi). $\mathrm{N}_{2}$ adsorption/desorption isotherms were measured using a surface area and porosity analyzer (ASAP2020, Micromeritics) with about $0.15 \mathrm{~g}$ of sample degassed at $120^{\circ} \mathrm{C}$ for 12 hours. Cumulative pore volume was calculated via the non-localized density functional theory (NLDFT) provided by Micromeritics. Cyclic voltammograms were recorded using a potentiostat (Reference 600, Gamry) attached to a three-electrode cell containing approximately $0.36 \mathrm{~cm}^{2}$ of sample working electrode, a saturated calomel reference electrode (SCE), and a $1.8 \mathrm{~cm}^{2}$ carbon counter electrode.

\subsection{Testing Using Stack A and B}

A flow-through CDI stack consisting of 16 electrode pairs, an in-line conductivity sensor (Alpha Cond 500, Thermo Scientific), an in-line pH sensor (Alpha pH 500, Thermo Scientific), a polyethylene tank, and a peristaltic pump (Masterflex L/S, Cole-Parmer) were assembled. CDI tests were performed at $1.2 \mathrm{~V}$ charging and $0 \mathrm{~V}$ discharging (1.2/0 V) in about $31 \mathrm{~L}$ of $7 \mathrm{mM}$ deaerated $\mathrm{NaCl}$ solution at $80 \mathrm{~mL} \mathrm{~min}^{-1}$. Two structurally identical CDI stacks with different anodes were used, and the construction of a CDI stack in detail can be found in ref. [17]. Stack A was configured with 16 pieces of the Pr-SC anodes and 16 pieces of the Pr-SC cathodes while Stack B was assembled with 16 pieces of the A-SC anodes and 16 pieces of the Pr-SC cathodes. 
A micrograph of the A-SC anode in Fig. 1(a) shows a combined anionic polymer layer with the Pr-SC carbon cloth. (The micrograph of the Pr-SC has been described in ref. [12]) The Pr-SC carbon cloth is overlaid with a graphite or titanium current collector to carry electric charges in both cyclic voltammetry and CDI tests. (See Fig. S8) As depicted in the inserted photograph, the prepared A-SC was cut to a geometric area of $\sim 7 \times 4 \mathrm{~cm}^{2}$ with the anionic polymer layer placed in the center. The A-SC anode including the $6.5 \times 3.5 \mathrm{~cm}^{2}$ painted area was paired with the $\sim 7 \times 4 \mathrm{~cm}^{2}$ Pr-SC cathode to configure a CDI cell in the present CDI tests. Additionally, 1 piece of the A-SC anode and Pr-SC cathode had average masses of $\sim 0.89$ and $\sim 0.38 \mathrm{~g}$, respectively.

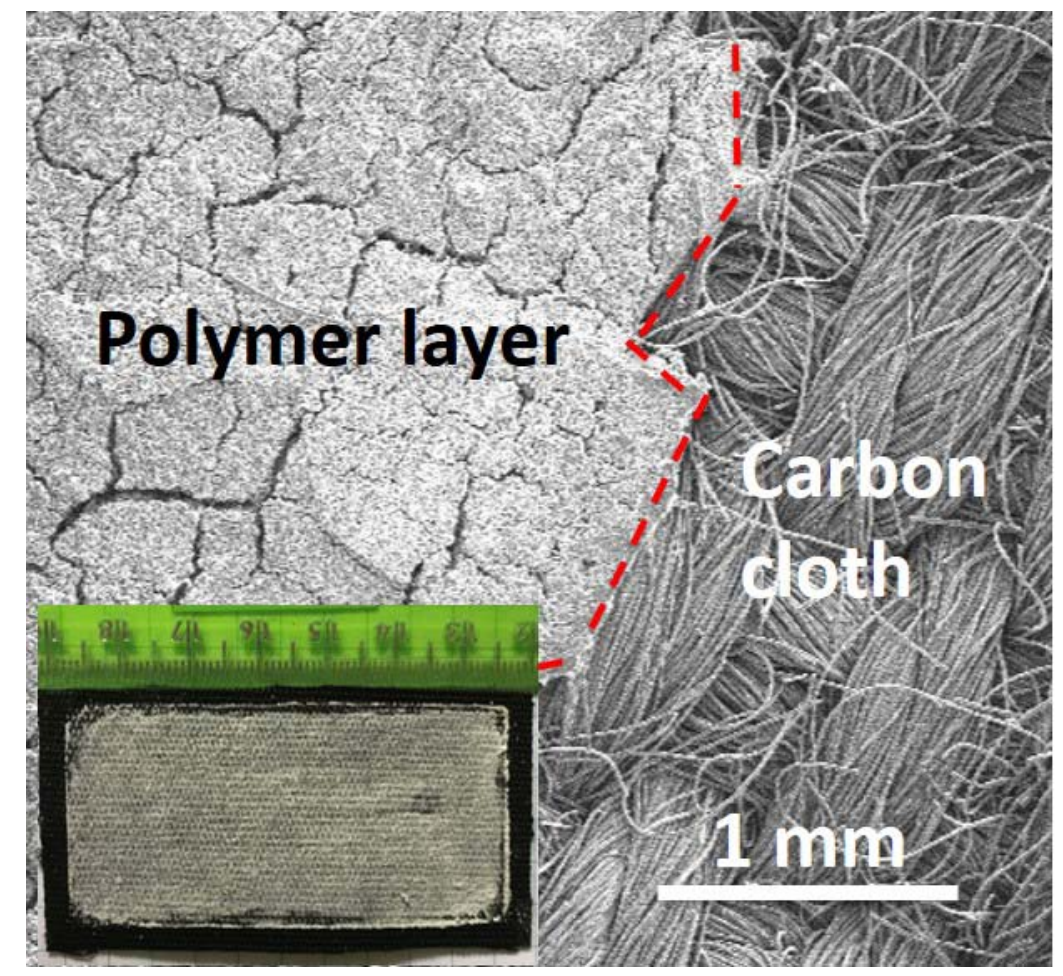

Fig. 1 Micrograph and photograph (inset) of the A-SC anode including a combined anionic polymer layer with the Pr-SC carbon cloth.

The total salt adsorption, SA, for Stack A and B was calculated by multiplying the 
volumetric flow rate, $\Phi$, by the integration of the concentration, $c$, with time, $t$, using

$$
\mathrm{SA}=(M \Phi) \int\left(c(\mathrm{t})-c_{\mathrm{in}}\right) d t
$$

where $M$ is the molecular weight of $\mathrm{NaCl}\left(58.44 \mathrm{~g} \mathrm{~mol}^{-1}\right)$ and $c_{\text {in }}$ is the influent concentration. The charge passed, $Q$, for the charging (or salt adsorption) step was calculated by integration of the current curves with time. Finally, the charge efficiency, $\Lambda$, is calculated using

$$
\Lambda=(\mathrm{SA} F) /(M Q)
$$

where $F$ is the Faraday's constant $\left(96,485 \mathrm{C} \mathrm{mol}^{-1}\right)$. Before $\mathrm{CDI}$ testing, the $\mathrm{NaCl}$ solution was circulated through both CDI stacks at open-circuit voltage until the effluent pH stabilized.
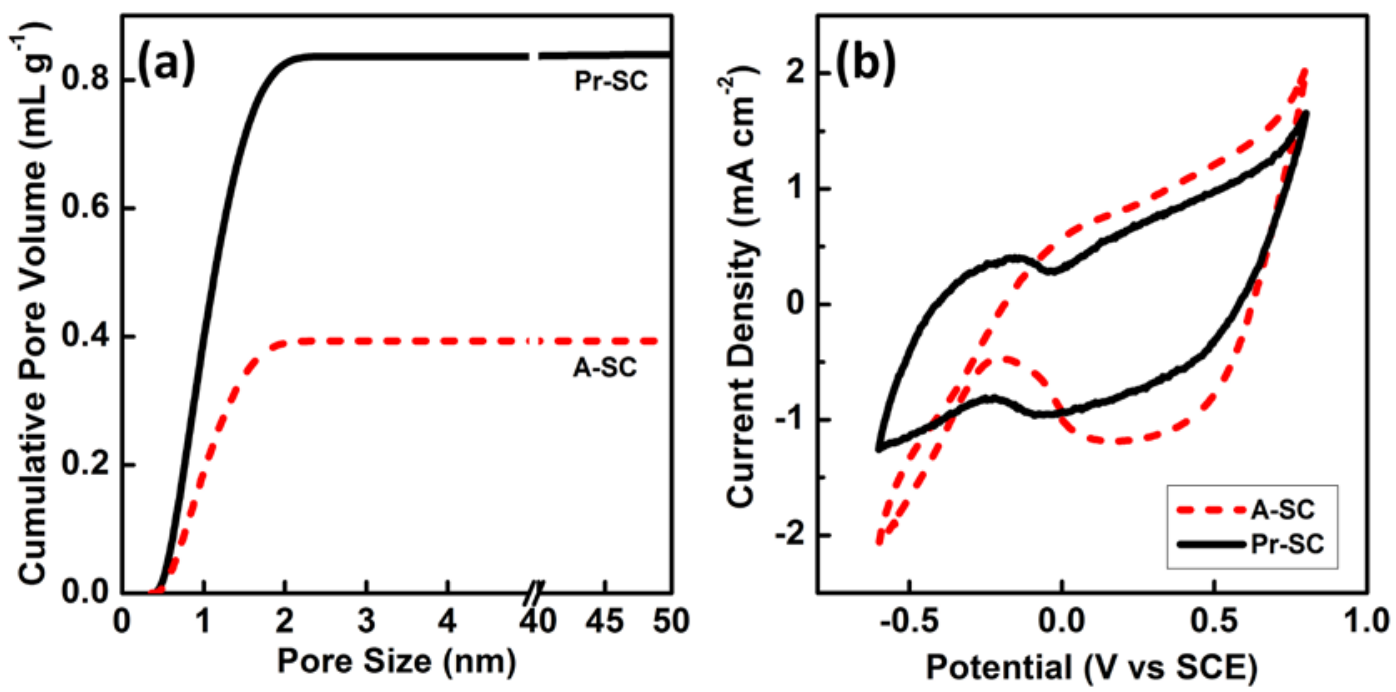

Fig. 2 Characterizations of the A-SC anode (dashed lines) and Pr-SC cathode (solid lines). (a) cumulative pore volume versus pore size resulting from $\mathrm{N}_{2}$ adsorption/desorption isotherms, and (b) cyclic voltammograms normalized by the 
geometric surface area, $0.36 \mathrm{~cm}^{2}$. Cyclic voltammetry was performed at $0.5 \mathrm{mV} \mathrm{s}^{-1}$ in 7 mM deaerated NaCl. (See Fig. S1-4 regarding additional characterizations and discussion.)

\section{Results and Discussion}

\subsection{Electrode Characterizations}

Cumulative pore volume versus pore size is plotted in Fig. 2(a). A sharp increase in the pore volume up to a pore size of $2 \mathrm{~nm}$ indicates that the Pr-SC is a microporous carbon, possessing a total pore volume of $0.84 \mathrm{~mL} \mathrm{~g}^{-1}$. By comparison, adding the polymer layer results in the total pore volume of the A-SC being reduced to $0.39 \mathrm{~mL} \mathrm{~g}^{-1}$, however the microporous carbon character is retained. As depicted in Fig. S1(b) and (c), the dried polymer solution (without being painted onto the Pr-SC) has no significant quantity of $\mathrm{N}_{2}$ adsorption/desorption in its isotherm, meaning that the impact of the polymer layer on the A-SC's total pore volume may be negligible. Therefore, the decreased pore volume of the A-SC in Fig. 2(a) is mainly attributed to the increased weight of the A-SC by the anionic polymer layer, which can be supported by comparing the capacitive behaviors in the corresponding voltammograms normalized by its total pore volume in Fig. S3(b).

As highlighted in Fig. 2(b), the cyclic voltammograms normalized by the geometric surface area of $0.36 \mathrm{~cm}^{2}$ show distinct V-shaped regions for both the Pr-SC and A-SC between -0.25 and $-0.04 \mathrm{~V}$ vs. SCE. At such potential corresponding to the $E_{\mathrm{PZC}}$, these electrodes have the least capability for ion adsorption.[18] It is also found that the capacitive behavior for the A-SC to its V-shape in the cathodic region is significantly 
diminished, which is due to limited cation adsorption that stems from having an anionic polymer layer over the A-SC. The capacitive behavior for the A-SC to its V-shape in the anodic branch is still as pronounced as the Pr-SC. Thus, we consider that the use of the A-SC anodes paired with the Pr-SC cathodes should be suitable for CDI applications.

In addition, we expect that the SA for Stack B (using the A-SC anodes) should be similar to that for Stack A, as the total pore volume for both of the stacks are almost equal, $\sim 10.2 \mathrm{~mL}$ for Stack A and $\sim 10.6 \mathrm{~mL}$ for Stack B, where the total pore volume in a CDI stack was calculated via the weight of the electrodes multiplied by the pore volume for an electrode depicted in Fig. 2(a). 

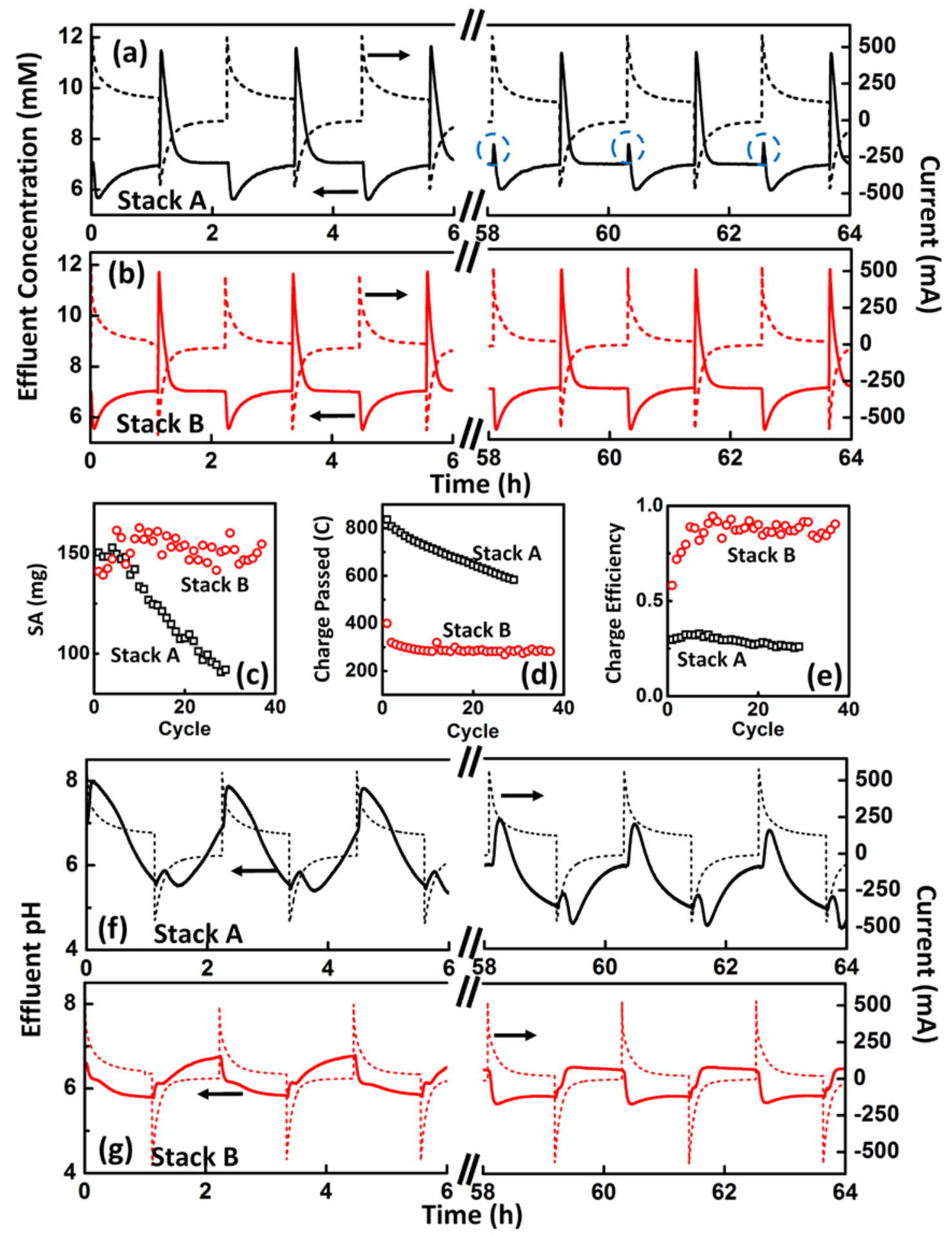

Fig. 3 CDI stack performance. (a) and (b) selected profiles of effluent concentration (solid lines) and current (dashed lines), (c) - (e) summary of charging step performance evaluations without normalization, and (f) and (g) selected profile of effluent $\mathrm{pH}$ (solid 
lines). Stacks A and B were repetitively tested at $1.2 / 0 \mathrm{~V}$ in $7 \mathrm{mM}$ deaerated $\mathrm{NaCl}$ solution at $80 \mathrm{~mL} \mathrm{~min}{ }^{-1}$. In addition, Stack A and B have total electrode weights (carbon and polymer) of about 12.3 and 20.5 g, respectively. (See the normalized plots in Fig. S5.)

\subsection{Testing}

In Fig. 3(a) and (b), for the first 6 hours of operation, both cell stacks show typical salt removal behavior, i.e., a decrease in the effluent concentration during charging. However, between the $58^{\text {th }}$ and $64^{\text {th }}$ hours of operation, Stack A displays a diminished effluent profile and desorption peaks during charging highlighted by dashed circles, while Stack B has maintained its initial performance. This undesirable salt desorption event indicates that salt removal in Stack A has been degraded without using the A-SC anodes. In Fig. 3(c) and (d), results evaluated based upon the profiles of the effluent concentration and current show that Stack A has a decreased SA from 151.3 to $92.3 \mathrm{mg}$ (of $\mathrm{NaCl}$ ) (12.3 to $7.5 \mathrm{mg} \mathrm{g}^{-1}$ ) accompanied by decreased charge passed from 836.4 to $583.0 \mathrm{C}$ (68.0 to 47.4 $\mathrm{C} \mathrm{g}^{-1}$ ), whereas Stack B maintains SA and charge passed at $155.8 \mathrm{mg}$ (of $\mathrm{NaCl}$ ) (7.6 mg $\mathrm{g}^{-1}$ ) and 285.0 C (13.9 C g $\left.{ }^{-1}\right)$, respectively, after 40 cycles. Correspondingly, in Fig. 3(e), during all tests, lower charge efficiency is observed for Stack A (28\%) compared to Stack B (85\%), which can be attributed to the greater steady-state (or leakage) current produced by Stack A. Additionally, in Fig. S5, if normalization is carried out using the total mass of the composite electrodes, i.e., carbon and polymer, Stack B will have a lower salt adsorption capacity than Stack A, since the anionic polymer layer itself does not aid in salt adsorption/removal. 
Besides the SA degradation interpreted in the introduction section or in ref. [3, 4], we consider that such differences in the charge efficiencies between Stack A and B are due to differences in their current responses in Fig. 3(a) and (b), for which the steadystate current during charging in Stack A ( 135 mA) is much greater than that in Stack B ( $25 \mathrm{~mA})$. The lower leakage current seen for Stack B may be attributed to the shielding of the electrodes by the polymer layers applied to the anode in the CDI stack. Steadystate currents (or leakage currents) can be indicative of the degree of charge transfer between electrode pairs and/or parasitic reactions, which can be further discussed by interpretation of the effluent $\mathrm{pH}$ for Stack A and B during charging.

In Fig 3(f) and (g), during the first few charging cycles at $1.2 \mathrm{~V}$, a decreasing effluent $\mathrm{pH}$ is observed, which is substantially more pronounced in Stack A than Stack B, suggesting that the overall process is dominated by reactions that can continuously produce $\mathrm{H}^{+}$. The thermodynamically most probable reactions accounting for leakage currents are water splitting, $\mathrm{H}_{2} \mathrm{O}_{2}$ production, dissolved oxygen reduction, and carbon oxidation.[19, 20] The predominant anodic reaction in our case is likely to be carbon oxidation via the following route(s): (1) $\mathrm{C}_{\text {(surface) }}+\mathrm{H}_{2} \mathrm{O} \rightarrow \mathrm{COH}_{\text {(surface) }}+\mathrm{H}^{+}+\mathrm{e}^{-}$, (2) $\mathrm{COH}_{\text {(surface) }}+\mathrm{H}_{2} \mathrm{O} \rightarrow \mathrm{COOH}_{\text {(surface) }}+2 \mathrm{H}^{+}+2 \mathrm{e}^{-}$, and (3) $\mathrm{COOH}_{\text {(surface) }} \rightarrow \mathrm{COO}_{\text {(surface) }}^{-}+$ $\mathrm{H}^{+}$. These reactions cause increased surface acidity of the carbon electrodes and thus yield substantive negative surface charge density at the anodes. Meanwhile, in order to balance the anodic reactions, based upon recent work in ref.[19], $\mathrm{H}_{2} \mathrm{O}_{2}$ is perhaps electrochemically-produced by consuming $\mathrm{H}^{+}$and/or $\mathrm{OH}^{-}$formation at the cathodes in Stack A, as an initial increase in the effluent pH is observed in Fig. 3(f). However, similar fluctuations in effluent $\mathrm{pH}$ are not displayed in Fig. 3(g) for Stack B, which suggests that 
carbon oxidation is mitigated at the A-SC anodes, consequently resulting in a deficiency in $\mathrm{H}^{+}$for $\mathrm{H}_{2} \mathrm{O}_{2}$ production. During subsequent discharging for both the stacks, the observed increase in the effluent $\mathrm{pH}$ may be affected by both the influent $\mathrm{pH}$ from the bulk solution and/or the reversibility of the parasitic reactions at both the anodes and cathodes.

As shown in the profiles between the $58^{\text {th }}$ and $64^{\text {th }}$ hours and in Fig. S5, Stack A apparently possesses an overall descending $\mathrm{pH}$ level while Stack B shows a relatively stable $\mathrm{pH}$ level, indicating higher accumulation of $\mathrm{H}^{+}$in the bulk solution in Stack A. This comparison emphasizes that $\mathrm{H}^{+}$production via electrochemical carbon oxidation at the anodes was more favored in Stack A than Stack B, thus resulting in both the steadystate current and charge passed to be higher in Stack A than Stack B.

\subsection{Used Electrode Characterizations}

To further support understanding of anode oxidation, the $E_{\mathrm{PZC}}$ for all the used electrodes were examined by cyclic voltammetry after CDI testing. As reported in the CDI literature, a positive shifting of the $E_{\mathrm{PZC}}$ results from enhanced negative surface charges via carbon oxidation.[6, 7, 21, 22] Indeed, in Fig. 4, by comparison, only the anode from Stack A clearly depicts the positive shifting of the $E_{\mathrm{PzC}}$ by $\sim 0.26 \mathrm{~V}$ relative to the Pr-SC, where the $E_{\mathrm{PZC}}$ is averaged based upon the $\mathrm{V}$-shapes associated with the anodic and cathodic branches. In contrast, the A-SC anode from Stack B shows virtually no $E_{\mathrm{PZC}}$ shift after prolonged cycling. Therefore, it can be concluded that the higher charge efficiency achieved in Stack B is due to the additional anionic polymer layer on the A-SC anode, effectively mitigating carbon oxidation and consequently preserving the A-SC's 
$E_{\text {PzC, }}$ which thus results in stable salt removal without forming inversion peaks in Fig. 3(b). Furthermore, we consider that competing processes such as carbon oxidation and anion adsorption at the anode always occur during charging in long-term CDI testing. Similar to ion-exchange membranes (or charge barriers) used in membrane-assisted capacitive deionization (MCDI) and CDI cells, $[8,13,23]$ adding an anion exchange/positively charged polymer layer to the anode selectively facilitates anion adsorption and may reduce the kinetics of parasitic oxidation by limiting the molecular transport of electrochemically active species across the barrier, thus resulting in more efficient and stable CDI operation. Additionally, based upon the knowledge gained in ref.[7, 8, 12] regarding enhanced CDI (e-CDI), we expect that this A-SC anode paired with cathodes containing negative surface charges can further boost the SA and charge efficiency, which will be presented in a future study.

Finally, based upon the unchanged voltammograms for the used cathodes in Fig. 4(c) and (d), at 1.2/0 V operation, parasitic reactions at the cathodes have much less impact on CDI salt removal compared to carbon oxidation at the anodes. Even though slightly decreased pore volume for all used samples are observed in Fig. S7, similar current densities in these voltammograms before and after CDI testing indicates that the electrodes' pore volumes available for salt adsorption have been generally maintained over the course of the testing period in the present study. 

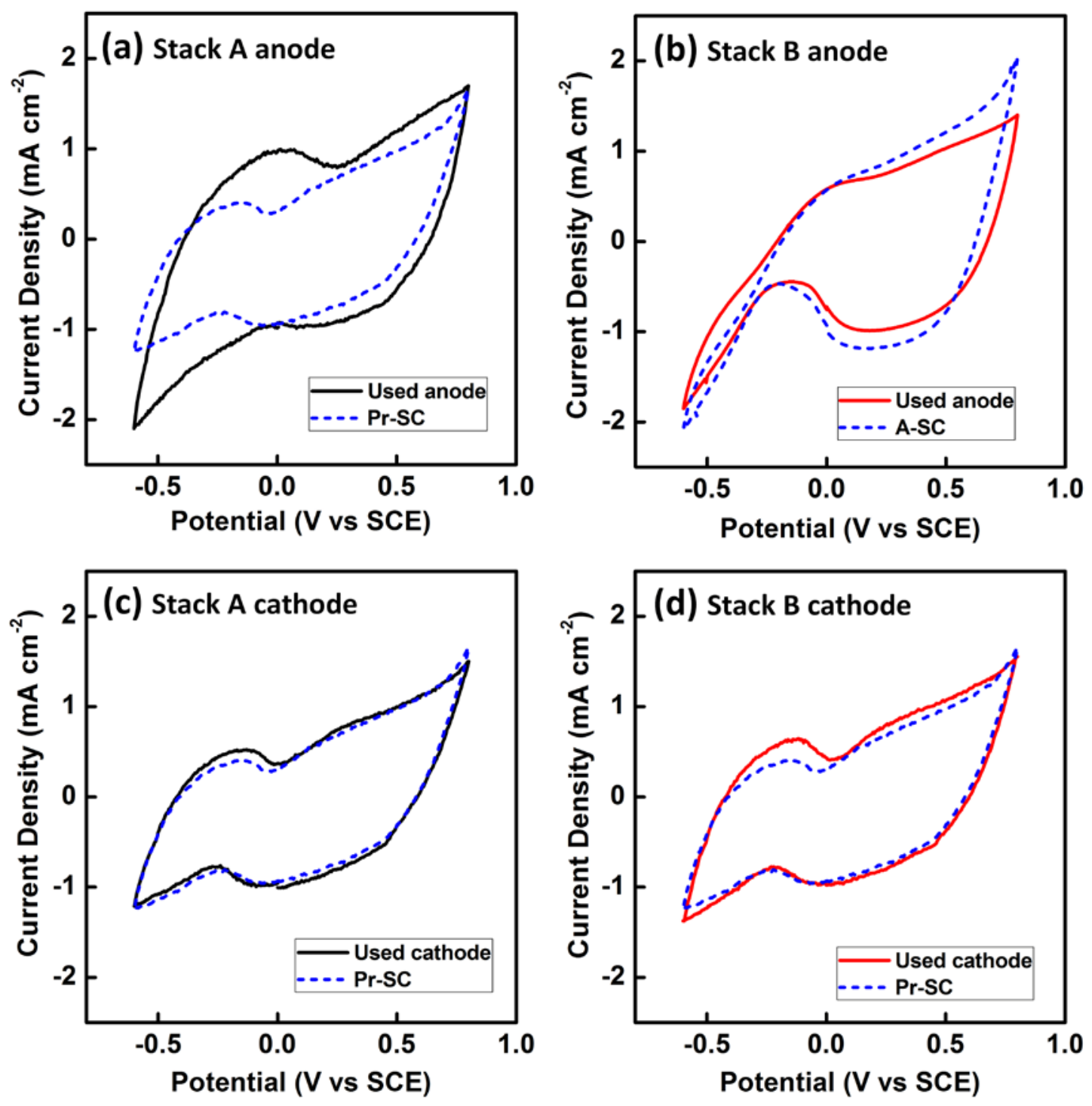

Fig. 4 Cyclic voltammograms for the carbon electrodes used in the first electrode pair in Stack A and B at $0.5 \mathrm{mV} \mathrm{s}^{-1}$ in $7 \mathrm{mM}$ deaerated $\mathrm{NaCl}$ solution. (a) and (b) initial and used anodes. (c) and (d) initial and used cathodes.

\section{Conclusion}

We prepared a composite carbon electrode with an anionic polymer layer for use as a CDI anode. Results from repetitive CDI tests showed more efficient and stable salt 
removal was achieved when a CDI stack was configured with composite carbon anodes.

This achievement was attributed to the fact that the anionic polymer layer could effectively minimize carbon oxidation and steady-state (or leakage) current, thus minimizing parasitic charge consumption and consequently boosting the charge efficiency. Furthermore, minimized carbon oxidation could retard positive shifting of the $E_{\text {PZC }}$ associated with the anode, thereby resulting in stable salt removal with inversion peaks not seen over the course of this study.

\section{Acknowledgements}

This work was supported by the U.S.-China Clean Energy Research Center, U.S. Department of Energy [DE-PI0000017]; and the National Science Foundation [1520226].

\section{References}

[1] J. Cotruvo, N. Voutchkov, J. Fawell, P. Payment, D. Cunliffe, S. Lattemann, Desalination technology: health and environmental impacts, CRC Press2010.

[2] M.E. Suss, S. Porada, X. Sun, P.M. Biesheuvel, J. Yoon, V. Presser, Water desalination via capacitive deionization: what is it and what can we expect from it?, Energy Environ. Sci. 8 (2015) 2296-2319.

[3] I. Cohen, E. Avraham, Y. Bouhadana, A. Soffer, D. Aurbach, Long term stability of capacitive deionization processes for water desalination: The challenge of positive electrodes corrosion, Electrochim. Acta 106 (2013) 91-100.

[4] X. Gao, A. Omosebi, J. Landon, K. Liu, Dependence of the Capacitive Deionization Performance on Potential of Zero Charge Shifting of Carbon Xerogel Electrodes during Long-Term Operation, $J$. Electrochem. Soc. 161 (2014) E159-E166.

[5] F. Duan, X. Du, Y. Li, H. Cao, Y. Zhang, Desalination stability of capacitive deionization using ordered mesoporous carbon: Effect of oxygen-containing surface groups and pore properties, Desalination 376 (2015) 17-24.

[6] T. Wu, G. Wang, Q. Dong, B. Qian, Y. Meng, J. Qiu, Asymmetric capacitive deionization utilizing nitric acid treated activated carbon fiber as the cathode, Electrochimi. Acta 176 (2015) 426-433.

[7] P.M. Biesheuvel, H. Hamelers, M. Suss, Theory of Water Desalination by Porous Electrodes with Immobile Chemical Charge, Colloids Interf. Sci. Commun. 9 (2015) 1-5.

[8] M.D. Andelman, Ionic Group Derivitized Nano Porous Carbon Electrodes for Capacitive Deionization, J. Mater. Sci. Chem.Eng. 2 (2014) 16-22.

[9] X. Gao, J. Landon, J.K. Neathery, K. Liu, Modification of Carbon Xerogel Electrodes for More Efficient Asymmetric Capacitive Deionization, J. Electrochem.Soc. 160 (2013) E106-E112.

[10] J. Yang, L. Zou, N.R. Choudhury, Ion-selective carbon nanotube electrodes in capacitive deionisation, 
Electrochim. Acta 91 (2013) 11-19.

[11] X. Gao, A. Omosebi, J. Landon, K. Liu, Enhancement of charge efficiency for a capacitive deionization cell using carbon xerogel with modified potential of zero charge, Electrochem. Commun. 39 (2014) 22-25.

[12] X. Gao, S. Porada, A. Omosebi, K.L. Liu, P.M. Biesheuvel, J. Landon, Complementary surface charge for enhanced capacitive deionization, Water Res. 92 (2016) 275-282.

[13] M.D. Andelman, Flow through capacitor basics, Sep. Purif. Technol. 80 (2011) 262-269.

[14] M.D. Andelman, G.S. Walker, Charge barrier flow-through capacitor, US6709560, 2004.

[15] M.D. Andelman, Polarized electrode for flow-through capacitive deionization, US20140346046, 2012.

[16] I. Cohen, E. Avraham, Y. Bouhadana, A. Soffer, D. Aurbach, The effect of the flow-regime, reversal of polarization, and oxygen on the long term stability in capacitive de-ionization processes, Electrochim. Acta 153 (2015) 106-114.

[17] X. Gao, A. Omosebi, J. Landon, K. Liu, Surface charge enhanced carbon electrodes for stable and efficient capacitive deionization using inverted adsorption-desorption behavior, Energy Environ. Sci. 8 (2015) 897-909.

[18] A.J. Bard, L.R. Faulkner, Electrochemical Methods - Fundamentals and Applications, 2nd ed., John Wiley \& Sons, New York, 2001.

[19] D. He, C.E. Wong, W. Tang, P. Kovalsky, T.D. Waite, Faradaic Reactions in Water Desalination by Batch-Mode Capacitive Deionization, Environ. Sci. Technol. Lett. (2016).

[20] A. Soffer, M. Folman, The electrical double layer of high surface porous carbon electrode, $J$. Electroanal. Chem. Interf. Electrochemi. 38 (1972) 25-43.

[21] E. Avraham, M. Noked, I. Cohen, A. Soffer, D. Aurbach, The Dependence of the Desalination Performance in Capacitive Deionization Processes on the Electrodes PZC, J. Electrochem. Soc. 158 (2011) P168-P173.

[22] H. Tobias, A. Soffer, The immersion potential of high surface electrodes, J. Electroanal. Chem. Interf. Electrochem. 148 (1983) 221-232.

[23] A. Omosebi, X. Gao, J. Landon, K. Liu, Asymmetric Electrode Configuration for Enhanced Membrane Capacitive Deionization, ACS Appl. Mater. Interf. 6 (2014) 12640-12649. 